\title{
Comparison of plasma and urinary concentrations of uric acid measured by the colorimetric phosphotungstate and enzymatic uricase methods
}

\author{
C. S. HIGGENS, I. K. MOSS, J. T. SCOTT \\ From the Kennedy Institute of Rheumatology and Charing Cross Hospital, London W6
}

\begin{abstract}
Accurate measurements of uric acid are essential for diagnosis of hyperuricaemia and to measure the effects of uricosuric drugs. Routine estimations of concentrations are commonly based on colorimetric methods. ${ }^{1}$ These are closely controlled and inexpensive methods using modern automated continuous flow techniques. There are, however, important sources of error, especially in urinary estimations.
\end{abstract}

The more accurate enzymatic assay uses specific uricase to oxidise uric acid to allantoin. Manual and semiautomated uricase methods have been described..$^{2}$ These methods are, however, time consuming and require specialist skill and equipment, making them unsuitable for routine hospital use. In 1969 the first fully automated enzymatic assay using continuous flow techniques was described by Steele. ${ }^{4}$ This needed two ultraviolet spectrophotometers working in parallel together with a large range of expensive equipment; this made it impractical for routine use. An automated uricase assay combines the specificity and sensitivity of the enzymatic method with the controlled conditions of the continuous flow autoanalyser. A method based on these principles is being developed to provide accurate routine determinations of uric acid in plasma and urine samples. ${ }^{5}$

We compared estimations of plasma and urinary uric acid measured routinely in this hospital by a colorimetric method with manual results from the uricase assay to ascertain whether significant differences could be detected. The manual and a new automated uricase assay were then compared in the measurement of plasma and urinary uric acid concentrations.
SUBJECTS, METHODS AND

\section{RES U LTS}

Plasma and 24-hour urine collections were obtained from unselected patients with normal creatinine clearance, over a six month period. All samples and standards were assayed for uric acid using all methods.

Colorimetric phosphotungstate method. Uric acid added to phosphotungstate in alkaline solution produces an intensely blue colour caused by an uncharacterised chromophore. The colour yield is directly related to the concentration of uric acid. The automated method has high reproducibility and facilitates a high standard of quality control. Errors in this method are due to the activity of non-specific chromagens in the samples and from the loss of uric acid which precipitates with protein, which is then removed by dialysis. This results in non-linearity between colour yield and uric acid concentration especially in urine where non-specific substances-for example, cystine and thiols--can contribute to total measured colour.

Enzymatic uricase methods. Uric acid is oxidised by uricase to allantoin. The disappearance of uric acid may be monitored by measuring the associated decrease in absorbance at $292 \mathrm{nmol} / \mathrm{l}$ in a spectrophotometer. This method has a high degree of specificity and sensitivity for the analysis of both blood and urine. Accuracy and precision are affected by sampling errors and spontaneous changes in sample turbidity causing variations in light scattering and apparent changes in ultraviolet absorbance. The automated uricase method used a continuous flow technique to present samples to the spectrophotometer, thus reducing sampling errors.
Estimations of plasma concentrations of uric acid by the phosphotungstate colorimetric method were a mean of $17 \%$ higher than the corresponding manual uricase assay values $(n=63 ; r=0.979)$. This difference varied in proportion to the concentration.

Estimations of urinary excretion of uric acid by the phosphotungstate colorimetric method were a mean of $48 \%$ higher than by the manual uricase assay $(n=30 ; r=0 \cdot 840)$. This difference was not proportional to the concentration.

There was an extremely good correlation of plasma and urinary concentrations of uric acid by the manual and automated uricase methods.

\section{COMMENT}

The phosphotungstate colorimetric method was inferior in accuracy to the enzymatic uricase method in the measurement of plasma and urinary uric acid concentrations.

For routine use the inexpensive colorimetric method could be used with a correction factor for plasma uric acid estimations. For accurate estimations of urinary uric acid, however, only the enzymatic uricase assay was reliable. The specificity of the uricase method was combined with continuous flow techniques in a new automated uricase assay. Results from this assay correlated well with those from the manual method. An automated uricase assay is at present being developed for routine hospital use (L Duncan, C S Higgens, P C Nicholas, unpublished data).

Further progress in detailed monitoring of kinetic reactions together with cheaper highly purified enzyme preparations should lead to greater accuracy and precision for 
routine hospital estimations of plasma and urinary uric acids by the enzymatic uricase method.

\section{References}

1 Henry R J. Clinical chemistry. New York: Harper and Row, 1966: 278.
2 Liddle L, Seegmiller J E, Laster L. The enzymatic spectrophotometric method for determination of uric acid. J Lab Clin Med 1959; 54: 903-13.

3 Simmonds H A. A method of estimation of uric acid in urine and other body fluid. Clin Chim Acta 1967; 15: 375-8.
4 Steele T H. An automated enzymatic spectrophotometric method for the determination of uric acid. Technical Bulletin of the Registry of Medical Technologists 1969; 39: 270-4.

\title{
Analysis of human plasma and urine purines using high performance liquid chromatography (HPLC)
}

\author{
H. J. R YLANCE, RUTHC. WALLACE, AND G. NUKI
}

From the Rheumatic Diseases Unit, Department of Medicine (WGH), University of Edinburgh, Northern General Hospital, Edinburgh EH5 $2 D Q$

The recent development of microparticulate, chemically bonded packing materials for high performance liquid chromatography (HPLC) has allowed the development of sensitive methods for the detection and quantification of purine bases and nucleosides in biological fluids. Using reverse phase HPLC methodology developed by Hartwick and others ${ }^{1}$ we undertook quantitative analyses of purine nucleosides and bases in normal human plasma and adapted these methods to obtain a qualitative profile of urine purine excretion products.

Instrumentation consisted of a Waters P/N 80060 modular system 1 chromatograph comprising twin pumps, solvent flow programmer, injection, $254 \mathrm{~nm}$ fixed wave absorbent detector, and M730 data module. The column used is a pre-packed Bondapak (C18) column consisting of a porous silica support with an octadecyl (C18) chemically bonded stationary phase (particle size $10 \mu \mathrm{m}$, column dimensions $3.9 \times 300$ $\mathrm{mm}$ ). A dry-packed precolumn of the same material (37-50 $\mu \mathrm{m}$ particle size) protects the main column. The solvent system employed is a linear gradient; $100 \% \quad 0.02 \mathrm{~mol} / 1$ phosphate buffer $\mathrm{pH} 5.6$ to $40 \%$ methanol/water $(60 \% \mathrm{v} / \mathrm{v})$ in 35 minutes at ambient

Table 1 Normal values ( $\mu$ mol/l) plasma constituents. Figures are means (SD)

\begin{tabular}{lcc}
\hline & Women $(n=16)$ & Men $(n=9)$ \\
\hline Creatinine & $92.6(17 \cdot 14)$ & $97 \cdot 8(12 \cdot 4)$ \\
Uric acid & $182.4(32 \cdot 9)$ & $297.5(64 \cdot 1)$ \\
Tyrosine & $44.7(21 \cdot 9)$ & $40.1(35 \cdot 8)$ \\
Hypoxanthine & $2.21(2.67)$ & $1.96(1.47)$ \\
Uridine/Xanthine & $2.63(1.05)$ & $2.94(0.98)$ \\
Inosine & $1.38(0.91)$ & $1.08(0.94)$ \\
Guanosine & \multicolumn{2}{c}{ Generally below limit of estimation } \\
Adenosine & $1.55(1.39)$ & $1.05(0.78)$ \\
\hline
\end{tabular}

temperature and a flow rate of $1 \cdot 5$ $\mathrm{ml} / \mathrm{min}$.

$40 \mu$ l plasma or $10 \mu$ l of a 1 in 10 dilution of urine are injected after ultra filtration in Amicon cones (CF 50A).

The identification of plasma purine and nucleoside peaks has been verified by cochromatography with pure compounds and enzyme shift methods. The table shows normal values for plasma constituents that may be accurately quantified. The lower limit for detection of plasma purines and nucleosides is approximately 0.2 $\mu \mathrm{mol} / \mathrm{l}$.

Urinary purine base and nucleoside concentrations are much more variable and dependent on diet and hydration. The definitive identification of all urine components detected has not been completed and many are partly excreted as methylated metabolites.

The urine chromatogram may, however, be used to provide a qualitative 'profile' of purine excretion in addition to quantitative measurements of uric acid and creatinine.

\section{Reference}

1 Hartwick R A, Krstulovic A M, Brown $P$ R. Identification and quantitation of nucleosides. bases, and other uv absorbing compounds in serum using reversed phase high performance liquid chromatography. II. Evaluation of human sera. J Chromatogr 1979; 186: 659-76. 